\title{
Android Based Low Cost Sitting Posture Monitoring System
}

\author{
Nusrat Binta Nizam ${ }^{1}$,Tohfatul Jinan ${ }^{2}$, Wahida Binte Naz Aurthy ${ }^{3}$, Md. Rakib Hossen ${ }^{4}$ and Jahid Ferdous ${ }^{5}$ \\ Department of Biomedical Engineering, \\ Bangladesh University of Engineering and Technology \\ Dhaka-1205, Bangladesh \\ Email: ${ }^{1}$ nusratbintanizam@ug.bme.buet.ac.bd, ${ }^{2}$ tohfatuljinan@ug.bme.buet.ac.bd, ${ }^{3}$ wahidabintenaz@ug.bme.buet.ac.bd, \\ ${ }^{4}$ mdhossen@ug.bme.buet.ac.bd, ${ }^{5}$ ferdousj@bme.buet.ac.bd
}

\begin{abstract}
Back pain is one of the leading causes of disabilityadjusted life year globally and the most common cause of low back pain is poor sitting posture. There are several actions that can be adopted proactively to avoid poor sitting posture induced back pain including behavioral change, regular exercise, and use of an ergonomic chair. However, these are either expensive and/or difficult to execute for prolonged periods. Sitting posture monitoring systems continuously observe the sitting pattern of a person in real-time and give feedback/alert poor sitting posture is observed. In this study, a real-time posture monitoring system has been designed and a functional prototype has been developed using simple electrical elements and an android application. Appropriate position of the sensor in the spine to measure the degree of bending and the threshold sensor values for good posture sittings have been determined based on the results from healthy volunteers of different ages and height. The android application continuously monitors the degree of bending and provides vibration when the bending reaches the threshold of bad posture or the duration of the sitting crosses the clinically recommended time limit to prevent prolonged sitting. Positive user feedback has been received in terms of comfortability, effectiveness, and satisfaction levels. The manufacturing cost of the developed monitoring system is minimal compared to the available expensive systems in the market and the cost would further go down if it is produced in bulk. This device efficiently monitors the sitting posture pattern to prevent back pain and within the affordable price range for the people from middle to under-developed countries.
\end{abstract}

Index Terms-Back pain; sitting posture monitoring system; android application; spine.

\section{INTRODUCTION}

Proper sitting posture refers to the position in which natural spinal curvature and arm, shoulder, hip, and knee position are properly maintained and aligned. Incorrect posture gradually decreases the flexibility of the spine causing pain in neck, shoulder and back. Furthermore, incorrect sitting posture has detrimental effects on digestion and respiration. Back pain is recognized as a common health issue and $75-85 \%$ of adults deal with back pain at least once in their lifetime [1]. In 2016, low back pain and neck pain combinedly were the $4^{\text {th }}$ leading cause of disability-adjusted life year (DALY) globally [2]. The World Health Organization (WHO) reported that 9.5 million people in South-East Asia were affected by back and neck pain. A descriptive cross-sectional study conducted among school teachers from different school in Dhaka showed $46 \%$ of school respondents have reported lower back pain [3]. Lack of knowledge of correct posture is assumed to be the primary cause of backache, yet a study that $60.8 \%$ of physiotherapists in Bangladesh have low back pain [4]. A cross-sectional study on Ready-Made Garment employees (RMG) of Bangladesh have reported $24.7 \%$ of female worker respondents had lower back pain and $23 . \%$ of female worker respondents had neck pain [5]. As many people spend most of the time sitting down in the workplace or home, bad alignment of sitting causes several problems such as bad support on muscles, ligaments, tendons and unhealthy spine [6].

There are several treatment methods for these abnormalities and depending on the severity, doctor advices to stay active and/or bed rest, prescribe medication [7], physical therapies [8], exercises [9], surgery [10], spinal traction therapy [11] etc. On the other hand, as a proactive practice, good sitting posture improves the body's circulatory and digestive system, increases self-confidence, decreases the risk of abnormal wearing of the joint surfaces, improves core and scapular strength etc [12]. Therefore, it is an excellent decision to make a habit of good sitting posture by monitoring as it brings many benefits for the human body without any unnecessary sideeffects. There has been a lot of effort to prevent back pain using eTextile sensor [13] and flex sensor to monitor sitting posture based ergonomic design. Moreover, there are some approaches based on machine learning and accelerometers [14] [15]. Most of these are very useful but complex, difficult to operate and very expensive.

The objective of this project is to develop a relatively inexpensive, comfortable and user-friendly system to monitor sitting posture in real-time. An elementary portable device connected to a smartphone via bluetooth using a flex sensor is represented. The user has to set the range in which he feels comfortable and receives a vibration in the motor when the angle crosses the safe range of good posture. 


\section{Design Methods}

\section{A. Overall Design}

The proposed design contains two parts: a circuitry part and a mobile application (app) part (Fig. 1). The circuitry part mainly determines the sitting posture pattern and sends signal to the app. The app monitors the duration of sitting and gives feedback to the user it the bending reaches the safety threshold limits.

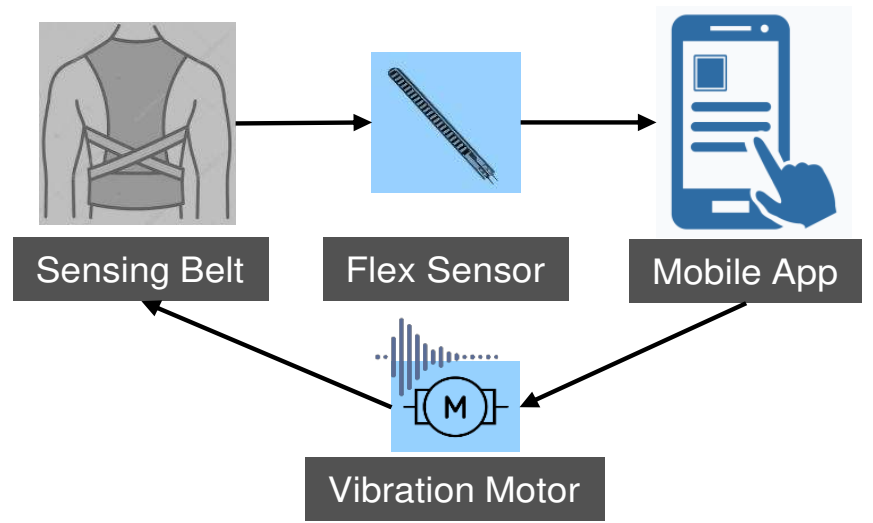

Fig. 1: Block diagram of the system.

\section{B. Instrumentation}

1) Materials: Detachable flex sensor (SEN-08606), Vibration Motor (Grove), Bluetooth (HC-05) device are used along with an Arduino-nano (Atmel ATmega 328) to operate and/or control. All of these electrical devices are assembled on a printed circuit board (PCB). A back supporting belt (Royal Posture) is used that holds the whole circuitry part. A $12 \mathrm{~V}$ rechargeable LIPO battery with average lifespan of 150-250 cycles is used as a power supply. The overall weight of the device including the battery is $340 \mathrm{gm}$.

2) Circuit Design: The circuit components are implanted on a 4-inch $x$ 4-inch PCB and 4-inch-long flex sensor whereas the battery and the vibration motor are attached to the sensor belt (Fig. 2). The circuit contains a voltage regulator system that converts the $12 \mathrm{~V}$ to $5 \mathrm{~V}$ to ensure the proper power supply. Bluetooth transfers the sensor value to the android app to set the threshold value based on the user comfortability and continuously monitors the posture position. The correlation between the sensor value and posture angle had been developed to convert sensor value corresponding to the user's postural angle. The vibration motor feedback system alerts the user to get back to the initial set postural position once the sitting posture angle crosses the threshold of good sitting posture.

3) Mobile APP Design: The home page of the mobile app named SIPO app has two options i.e., "Connect to Arduino" and "Instructions". Instruction window illustrates a tutorial about the proper sitting process for the user (Fig. 3). The user has to connect the app with the Arduino in PCB through the connect option. After connecting the device, the user has to set a threshold value from the sensor reading on the app screen

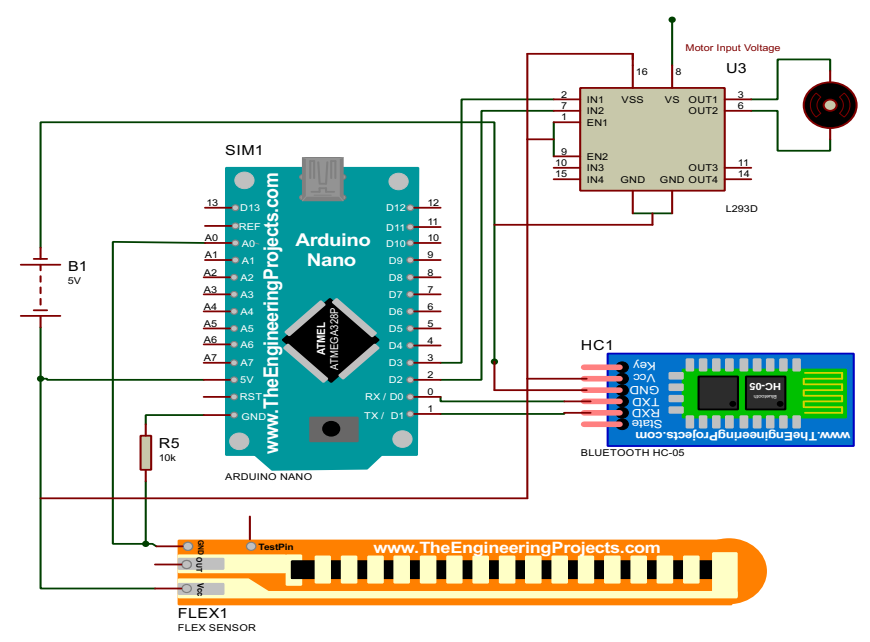

Fig. 2: Circuit connections in proteus software.

according to his comfortable position to monitor posture at a fixed position continuously. The timer feature is for counting the total duration of sitting.

\section{Study Design}

An experimental study is done on healthy volunteers having a range of age, weight, height and gender to find the best position for the sensor placement, to calibrate the sensor value with posture angle and to get user feedback of the system to ensure the participant's safety and effectiveness.

1) Settings: A marker is used to observe and track the volunteers bending status when he is advised to bend forward and backward freely from a normal sitting position and a camera is used to capture the position for the angle measurement with respect to the initial position (Fig. 4). The mobile app (SIPO) needs to be installed and the circuitry part is used to observe the corresponding sensor value of the posture angle. ImageJ software/ angle meter app is used to calculate the angle from the images. It is important to make sure that there are enough spaces so that the user can easily move forward and backward while sitting.

2) Data Collection from Participants: Four positions are selected to specify the best location for the sensor placement based on the average human anatomical position to best track the bending movement. They are- (a) upper thoracic $(6 \mathrm{~cm}$ below neck), (b) lower thoracic (12 cm below neck), (c) upper lumbar $(20 \mathrm{~cm}$ below neck), and (d) lower lumbar $(26 \mathrm{~cm}$ below neck). In order to develop the relationship between the sensor value and posture angle, participants are advised to change their posture angle by moving forward and backward six times (three times each side covering a wide range of posture angle) from sitting at a normal position. The position of the sensor is an important factor as it varies with the height of the person. There are in total 9 participants, age ranging from 21 to $50( \pm 7.91)$, height and weight ranging from 5' to 5'11" $( \pm 0.29)$ and $45 \mathrm{~kg}$ to $75 \mathrm{~kg}$ respectively. 


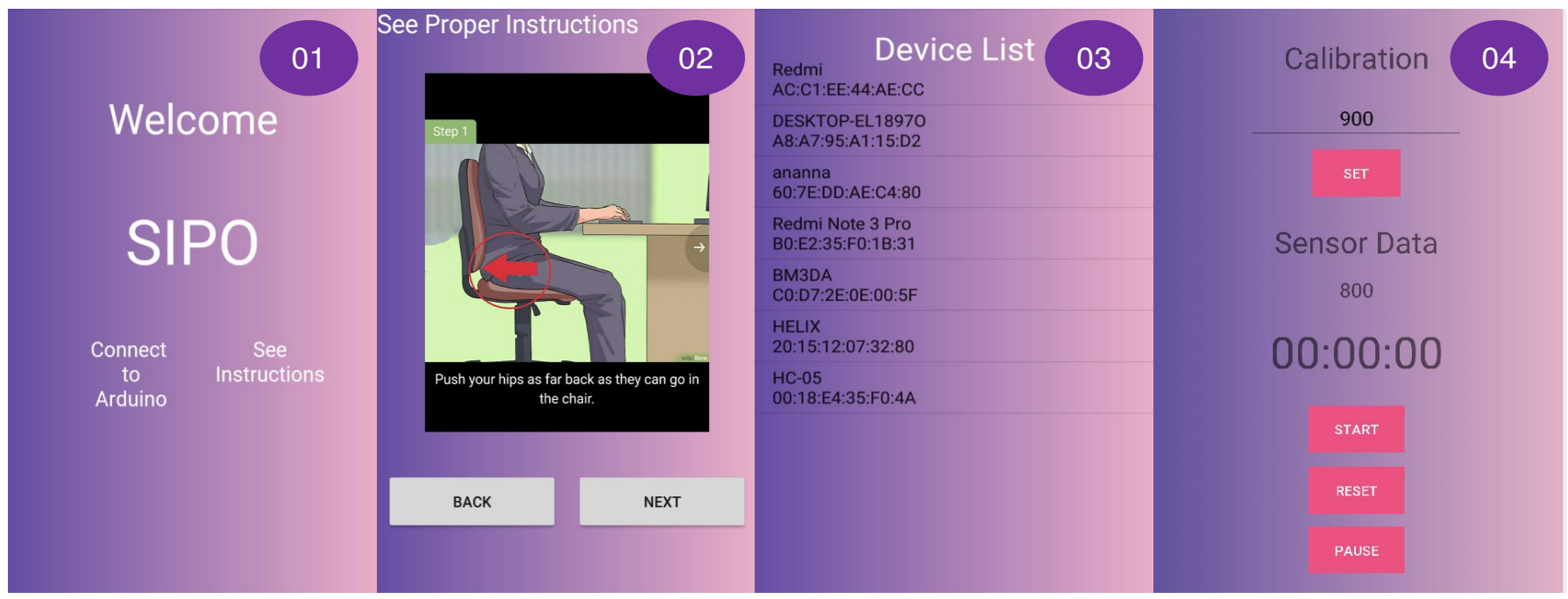

Fig. 3: Pages of android application, where, (1) Home page, (2) Instruction page, (3) Connection page, and (4) Monitoring and timer tab.
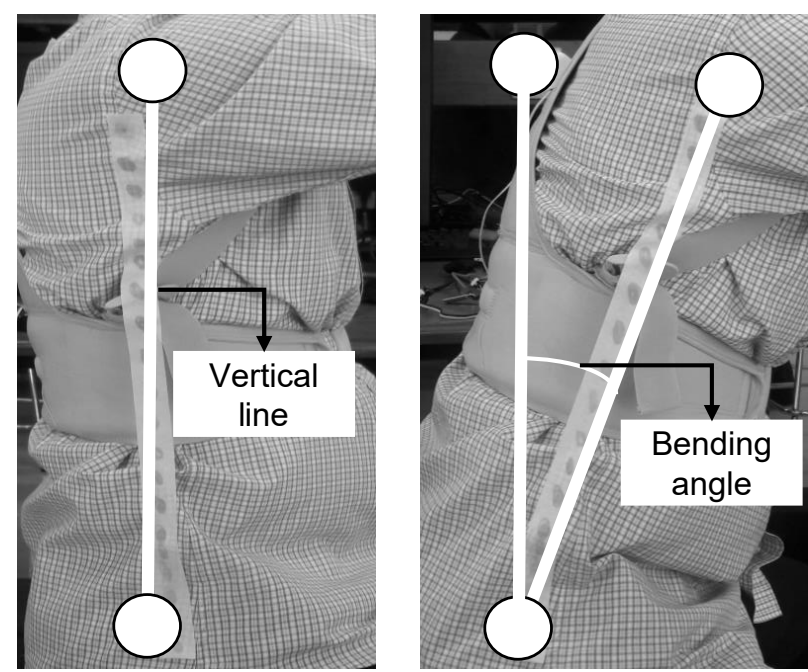

Fig. 4: Posture bending angle measurement.

\section{User Feedback}

User feedback form has been filled by the user to evaluate the different aspects of the device from end-user point of view (comfortability, preference, effectiveness, satisfaction).

\section{RESULTS ANALYSIS}

\section{A. Position Variability Analysis}

For the analysis of the sensor position variability, the collected data of four different positions are studied at five different selected angles $\left(75^{\circ}, 80^{\circ}, 90^{\circ}, 100^{\circ}, 115^{\circ}\right)$. For a fixed position and angle value, the sensor values of 9 subjects are averaged (Fig. 5). In the lower thoracic position the range of sensor values for different angles is higher, which shows better sensitivity of sensor and wide range of sensor values at this position. For this reason, the lower thoracic position is selected for the placement of the sensor.

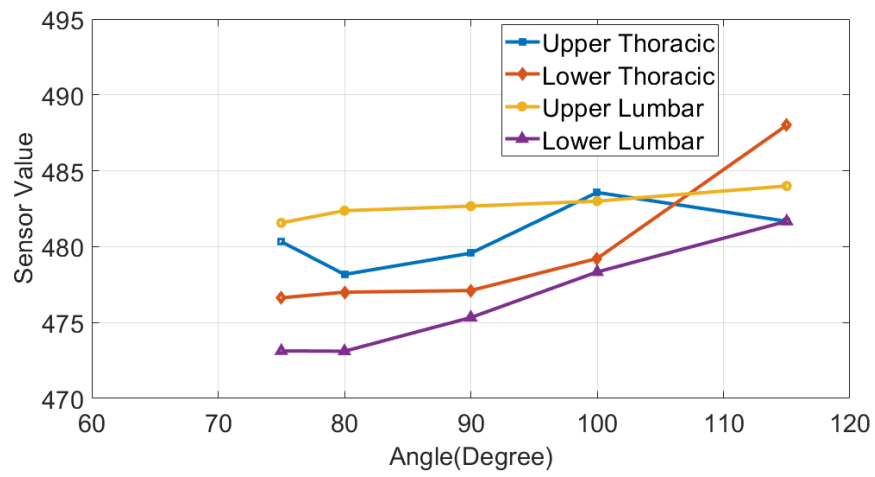

Fig. 5: Determination of the sensor position for the best sensitivity.

\section{B. Threshold Range Calculation}

Bending angle range of $90^{\circ}-110^{\circ}$ has been reported as the safe zone for good posture whereas $90^{\circ}-95^{\circ}$ is the range of normal sitting posture (Fig. 6) [16]. Therefore, in order to develop the corresponding sensor value for good posture position, a model has been developed for lower thoracic position and fitted with a $3^{r d}$ degree of a polynomial equation (Eqn. 1). So, sensor value beyond the thresholds of safety regions generates signal to the vibration motor and alert the user to change the sitting position.

$$
S V=0.0003(A)^{3}-0.0605(A)^{2}+4.8789(A)+345.23(1)
$$

where $\mathrm{SV}=$ sensor value and $\mathrm{A}=$ angles in degrees. 


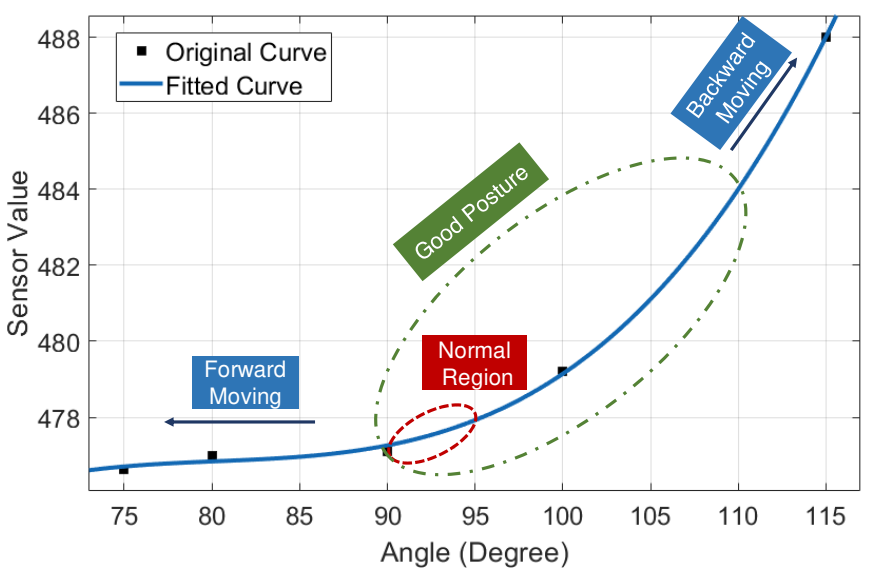

Fig. 6: Calibration between the sensor value and bending angle.

\section{DISCUSSION}

The survey on the users' feedback gets 7.6/10 in terms of comfortability, 9.2/10 in terms of preference, 9.6/10 effectiveness and 8.0/10 in terms of overall satisfaction based on the average of the given marks by the users in user feedback form. The feedback also indicates that the user will be able to use this device for 5-6 hours a day.

Continuous monitoring of a posture position through the developed app makes the device more used-friendly, comfortable, portable along with low-cost compared to the other devices available in the market. The available devices in the market cost about 150 USD - 600 USD, whereas the cost of SIPO is only about 42 USD. The manufacturing cost can be reduced by mass production thus making it an affordable solution for those who prefer to continuously monitor their sitting posture and achieve a healthy spine.

Sensitivity and accuracy of the system can be upgraded in future by controlled movement of bending during the experiment using more sophisticated camera and automatic calculation of the bending angle. More data from a large population having wide variation of height, weight, and age can be used to calibrate the sensor value with the bending angle. Moreover, the apps can be updated by adopting artificial intelligence and determine the initial set position and threshold values based on the user inputs (weight, age, gender, and height).

\section{CONCLUSION}

The primary goal of this project is to provide an effective solution to maintain a healthy sitting posture that is userfriendly, wearable, safe, inexpensive and non-invasive. The developed functional prototype demonstrates a cost-effective way for real-time monitoring of a posture position. Due to light-weight and portability, this wearable product is one of the best solutions for the desk-workers, students and travelers who spend a significant amount of time in sitting position.

\section{ACKNOWLEDGMENT}

This work has been conducted with the help of Department of Biomedical Engineering, Bangladesh University of Engineering and Technology. The authors would like to thank the volunteers for their cooperation. Special thanks to Navid Ibtehaj Nizam, Assistant Professor, Department of Biomedical Engineering, Bangladesh University of Engineering and Technology.

\section{REFERENCES}

[1] Y. Tanaka, "Epidemiology of low back pain," Clin. calcium, vol. 15, no. 3, pp. 35-38, 2005.

[2] S. I. Hay, A. A. Abajobir, K. H. Abate, C. Abbafati, K. M. Abbas, F. Abd-Allah, R. S. Abdulkader, A. M. Abdulle, T. A. Abebo, S. F Abera et al., "Global, regional, and national disability-adjusted lifeyears (DALYs) for 333 diseases and injuries and healthy life expectancy (HALE) for 195 countries and territories, 1990-2016: a systematic analysis for the global burden of disease study 2016," The Lancet, vol. 390, no. 10100, pp. 1260-1344, 2017.

[3] J. Akter, M. Kamrujjaman, and A. Maleque, "Prevalence and factors associated with low back pain among school teachers residing in northern dhaka city," 2018.

[4] R. Mondal, R. C. Sarker, S. Akter, P. C. Banik, and S. K. Baroi, "Prevalence of low back pain and its associated factors among physiotherapists in dhaka city of bangladesh in 2016," J. of Occupational Health and Epidemiology, vol. 7, no. 2, pp. 70-74, 2018.

[5] M. D. Hossain, A. Aftab, M. H. Al Imam, I. Mahmud, I. A. Chowdhury, R. I. Kabir, and M. Sarker, "Prevalence of work related musculoskeletal disorders (WMSDs) and ergonomic risk assessment among readymade garment workers of bangladesh: A cross sectional study," PloS one, vol. 13, no. 7, p. e0200122, 2018.

[6] Southern California Orthopedic Institute - in Alliance with UCLA Health, Regents of The University of California, 2020 (accessed July 18, 2020). [Online]. Available: https://www.scoi.com/services/ physical-therapy/importance-good-posture

[7] B. Koes, M. Van Tulder, and S. Thomas, "Diagnosis and treatment of low back pain,” Bmj, vol. 332, no. 7555, pp. 1430-1434, 2006.

[8] J. Rajfur, M. Pasternok, K. Rajfur, K. Walewicz, B. Fras, B. Bolach, R. Dymarek, J. Rosinczuk, T. Halski, and J. Taradaj, "Efficacy of selected electrical therapies on chronic low back pain: a comparative clinical pilot study," Med. Sci. monitor: Int. Med. J. of Exp. and Clin. Res., vol. 23, p. 85, 2017.

[9] Z. Abdollahzade, A. Shadmehr, K. Malmir, and N. Ghotbi, "Effects of 4 week postural corrective exercise on correcting forward head posture," J. of Modern Rehabil., pp. 85-92, 2017.

[10] K.-J. Cho, Y.-T. Kim, S.-h. Shin, and S.-I. Suk, "Surgical treatment of adult degenerative scoliosis," Asian Spine J., vol. 8, no. 3, p. 371, 2014.

[11] H. Oh, S. Choi, S. Lee, J. Choi, and K. Lee, "The impact of manual spinal traction therapy on the pain and oswestry disability index of patients with chronic back pain," J. of Physical Therapy Sci., vol. 30, no. 12, pp. 1455-1457, 2018.

[12] 12 Benefits of Good Posture - and How to Maintain It, 2020 (accessed July 18, 2020). [Online]. Available: https://www.healthline.com/health/ fitness-exercise/posture-benefits

[13] W. Xu, Z. Li, M.-C. Huang, N. Amini, and M. Sarrafzadeh, "ecushion: An etextile device for sitting posture monitoring," in 2011 Int. Conf. on Body Sensor Netw. IEEE, 2011, pp. 194-199.

[14] J. Roh, H.-j. Park, K. J. Lee, J. Hyeong, S. Kim, and B. Lee, "Sitting posture monitoring system based on a low-cost load cell using machine learning," Sensors, vol. 18, no. 1, p. 208, 2018.

[15] W. Y. Wong and M. S. Wong, "Detecting spinal posture change in sitting positions with tri-axial accelerometers," Gait \& Posture, vol. 27, no. 1, pp. 168-171, 2008.

[16] Proper Sitting Posture at a Computer (According to Experts)Ergonomic Trends, 2020 (accessed July 18, 2020). [Online]. Available: http://ergonomictrends.com/proper-sitting-posture-computer-experts/ 